\title{
Avaliação das características da contenção intra-radicular e tratamentos endodôntico em radiografias periapicais de 1000 dentes
}

\author{
Evaluation of the characteristics of the posts and cores and endodontic treatment in \\ periapical radiographs of 1000 teeth
}

\section{Inês Laluci DURIGHETTO}

Mestre - Universidade Federal de Uberlândia - UFU - Uberlândia - MG - Brasil

João Carlos Gabrielli BIFFI

Doutor - Universidade Federal de Uberlândia - UFU - Uberlândia - MG - Brasil

\author{
Antônio Francisco DURIGHETTO JÚNIOR \\ Doutor - Universidade Federal de Uberlândia - UFU - Uberlândia - MG - Brasil
}

Cristiane Melo CARAM

Cirurgiã-dentista

\begin{abstract}
Resumo
A proposta deste trabalho foi avaliar radiograficamente 1000 dentes tratados endodonticamente e restaurados com contenção intra-radicular, analisando a qualidade do tratamento, relacionando os com a presença de alteração apical. As imagens radiográficas foram digitalizadas e, então, feita a análise, que teve como critério de tratamento endodôntico satisfatório, quando canais obturados de $1 \mathrm{a} 2 \mathrm{~mm}$ aquém do ápice radicular, massa obturadora homogênea e material obturador remanescente de no mínimo $3 \mathrm{~mm}$. O pino foi considerado satisfatório, quando ocupava 2/3 do remanescente dental ou no mínimo o comprimento da coroa clínica, e o diâmetro apresentasse $1 / 3$ do diâmetro da raiz. O Espaço entre o pino e tratamento endodôntico, a descentralização do pino em relação ao longo eixo da raiz, a trepanação e a fratura radicular, foram considerados como pinos insatisfatórios. Lesões periapicais foram identificadas quando da presença de espessamento ou de área radiolúcida no ápice radicular. Ao final deste estudo, foi possível observar a relação, estatisticamente significante, entre tratamentos endodônticos insatisfatórios e lesão periapical, porém não foi observada esta relação entre pino insatisfatório e lesão periapical.
\end{abstract}

\section{UNITERMOS}

Endodontia; técnica para retentor intra-radicular.

\section{INTRODUÇÃO}

A realização do tratamento com prótese fixada a pino intra-radicular é muitas vezes o procedimento indicado, deve atender a princípios básicos de endodontia, periodontia, prótese laboratorial e prótese clínica, pois qualquer falha pode levar ao insucesso.

O tratamento endodôntico deve ser realizado dentro de princípios criteriosos, Ingle ${ }^{12}$ (1996), mostrou como causa maior dos fracassos $(63,46 \%)$ para os dentes tratados endodonticamente os fatores rela- cionados à percolação, dos quais 58,66\% referem-se à obturação endodôntica incompleta. Dentes cujo limite de obturação não se encontra entre 1 a $2 \mathrm{~mm}$ normalmente apresentam um baixo índice de sucesso no tratamento, isto pode ser observado em um estudo radiográfico de 898 dentes humanos com tratamento endodôntico, onde relacionaram o limite de obturação dos canais com a presença de alteração apical, considerando o limite ideal entre 1 a $2 \mathrm{~mm}$, e encontraram 
$54,35 \%$ de canais parcialmente obturados e, desses, $66,25 \%$ apresentaram alteração apical, demonstrando a estreita relação entre limite apical inadequado com presença de alteração apical ${ }^{9}$. Leonardo ${ }^{15}$ (1973), em estudo histológico de dentes humanos extraídos, analisou os ápices cujos canais haviam sido obturados a $0,5 \mathrm{~mm}$ aquém do ápice radiográfico e observou que, em muitos casos, havia ocorrido a sobre-obturação, ou seja, o material obturador tinha ultrapassado o limite apical. $\mathrm{O}$ autor atribuiu o achado ao fato de que o forame abre-se antes do vértice radiográfico. De Deus ${ }^{4}$ (1986) escreveu, em seu livro, que é aconselhável levar a obturação hermética do canal radicular a um limite apical de 0,5 a $1 \mathrm{~mm}$ aquém da superfície mais externa da raiz dentária, o que oferece maior segurança ao tratamento, diminuindo ou evitando as reações pós-operatórias imediatas e tardias. Isto somente não seria aplicado em algumas variações da normalidade. Tamburus $^{29}$ (1983), analisando radiografias periapicais, detectou que o índice de insucesso do tratamento endodôntico, indicado pela presença de alterações apicais, estaria relacionado, em 74,41\%, com obturações incompletas e, em 33,52\%, com a presença de porosidades na massa obturadora. Afirmou, ainda, que esse insucesso estaria também relacionado à presença de alterações apicais prévias que representou praticamente 55,8\%. Holland, Valle, Taintor e Ingle ${ }^{8}$ (1983), em estudo radiográfico comparativo mostraram que os tratamentos endodônticos, realizados em dentes que já apresentavam rarefação óssea apical antes do tratamento, obtiveram índices menores de sucesso do que aqueles nos quais não foi possível observar alterações apicais. Sjögren, Hagglund, Sundqvist e Wing $^{27}$ (1990) selecionaram e analisaram clínica e radiograficamente, 65 dentes que haviam recebido tratamento endodôntico há um período de 8 a 10 anos e demonstraram que as maiores dificuldades encontradas para obter sucessos terapêuticos estariam relacionadas a dentes cujo pré-operatório apresentava necrose pulpar, lesões periapicais e àqueles que haviam recebido retratamento endodôntico.

Outro fator importante no sucesso do tratamento com retenção intra-radicular está relacionado ao remanescente de material obturador deixado quando do preparo do espaço para colocação do pino. Caputo e Standlee $^{3}$ (1976) recomendaram que, após o preparo do espaço para o pino em um canal radicular tratado endodonticamente, um mínimo de $3 \mathrm{~mm}$ de obturação radicular deve ser mantido. Kvisk, Rydin e Reit ${ }^{14}$ (1989) comparando a frequiência de lesões apicais em dentes portadores de prótese com pino intrarradicular e remanescente de obturação de 2 e de 3 a $5 \mathrm{~mm}$, encontraram diferença significante, quando da comparação do remanescente obturador menor que $3 \mathrm{~mm}$ com o de 3 a $5 \mathrm{~mm}$, sendo a maior freqüência para radiolucência apical nos dentes com remanescentes inferiores a 3mm. DeCleen ${ }^{5}$ (1993), em uma revisão da literatura, recomendou um remanescente de material obturador após preparo de espaço para pino preferencialmente de $6 \mathrm{~mm}$, sendo tolerável de $3 \mathrm{~mm}$. Raiden e Gendelman ${ }^{21}$ (1994) realizaram pesquisa "in vitro", usando 67 blocos de resina acrílica transparente, simulando canais radiculares para observar o efeito da preparação do espaço para o pino no selamento apical de obturações de canais radiculares. As amostras foram divididas em 4 grupos, sendo que, nos grupos de $n^{\circ} 1,2,3$ e 4 , a remoção da obturação foi feita, tentando deixar $1 \mathrm{~mm}, 2 \mathrm{~mm}, 3 \mathrm{~mm}$ e $4 \mathrm{~mm}$, respectivamente, de material obturador. Após a presa do material e a imersão das amostras em azul de metileno por 72 horas, foram observados diferentes comprimentos de infiltração para os vários grupos analisados. Desta forma, concluíram que o preparo do canal para recebimento do pino não altera o selamento, desde que um remanescente de, no mínimo, $3 \mathrm{~mm}$ seja mantido. Quando do processo de restauração com prótese fixada a pino é muito importante o cuidado durante a cimentação no canal radicular. Esse procedimento não deve deixar espaços vazios entre o pino e as paredes laterais do canal radicular, dando proteção adequada na entrada dos canais, bem como entre a sua extremidade apical e o remanescente do tratamento endodôntico. Esses espaços vazios podem favorecer a infiltração de saliva e microorganismos o que aumentaria o risco de insucesso do tratamento ${ }^{1}$. O comprimento e diâmetro dos pinos são considerados fator importante para o sucesso de uma restauração com contenção intra-radicular. Stern e Hirschfeld ${ }^{28}$ (1973) analisaram os princípios do preparo para restaurações a pino em dentes tratados endodonticamente e concluíram que o comprimento do pino não só deveria ser maior que a coroa clínica, mas também deveria considerar a estrutura óssea que envolve o dente. A extensão apical do pino deveria estar localizada, no mínimo, na metade do comprimento entre o ápice da raiz e a crista óssea alveolar. Em relação ao seu diâmetro, estabeleceram que este deveria apresentar $1 / 3$ do diâmetro da raiz e, no terço apical, ter pelo menos $1 \mathrm{~mm}$ para proporcionar maior resistência à fratura. Selby ${ }^{25}$ (1994) revisou trabalhos de diversos autores sobre as possíveis causas de falências das próteses fixas e verificou que colocação de pinos de comprimento curto e diâmetro fino foram 
responsável pelo insucesso de muitos dentes tratados endodonticamente e retidos a pino. Morgano ${ }^{18}(1996)$ recomendou, em seu trabalho, que, quando houvesse a necessidade de um pino para restaurar um dente tratado endodonticamente, seu o comprimento não deveria comprometer o remanescente apical de guta-percha, que deveria ser mantido em 4 a $5 \mathrm{~mm}$, e sua largura não deveria exceder a metade do diâmetro da raiz. Quando não atendidos esses fundamentos, o sucesso do tratamento seria considerado baixo.

\section{Material e mÉtodo}

\section{Seleção das radiografias}

Na presente pesquisa foram utilizados 786 tomadas radiográficas periapicais, que permitiu o exame de 1000 dentes, de pacientes que procuraram um centro de radiologia da cidade de Uberlândia, para a realização de exame radiográfico com radiografias periapicais, como complemento de diagnóstico.

Os pacientes foram encaminhados por profissionais da cidade de Uberlândia e região.

As tomadas radiográficas selecionadas apresentavam um ou mais dentes permanentes restaurados, com contenção intra-radicular e tratamento endodôntico.

Não houve exclusão de dentes, e não foi feita a distinção entre idade e gênero dos pacientes, bem como a técnica de tratamento realizada.

\section{Obtenção das radiografias}

As tomadas radiográficas foram realizadas com aparelho de $70 \mathrm{Kvp}, 9 \mathrm{~mA}$ e área focal de $2,3 \mathrm{~mm}^{2}$ (Gnatus - TIMEX 70), alimentação nominal 220V, $60 \mathrm{~Hz}$. Foram utilizados posicionadores tipo HanSin, para a técnica do paralelismo (Prisma) e o filme ra- diográfico do grupo $\mathrm{F}$ e tamanho 2 (Kodak Insight - Eastman Kodak, Rochester, NY, USA).

O processamento foi realizado de forma padronizada, utilizando uma processadora automática com ciclo longo de 4 minutos (Gendex-Gxp made in USA modelo-110-0096-gi - MGF.Série-40011236162DF date dezembro 1994 (120 volts - PHSE1-Wz 60).

\section{Análise das radiografias}

As imagens radiográficas selecionadas foram digitalizadas, possibilitando a melhor definição de contraste e brilho e, ainda, a inversão da imagem para o negativo, quando fosse necessária uma melhor definição. As raízes selecionadas para julgamento dos dentes multirradiculares foram, palatinas de molares superiores e distais dos molares inferiores e palatinas dos pré-molares superiores.

A análise foi feita por um único observador, especialista em imaginologia odontológica. Ao final das análises radiográficas, foi feita uma classificação do tratamento endodôntico e do pino em satisfatórios ou insatisfatórios e do ápice em com lesão e sem lesão.

\section{Resultados}

De acordo com as análises feitas, observou-se que, dos 1000 dentes analisados radiograficamente, 774 $(77,4 \%)$ apresentavam tratamento endodôntico insatisfatório e $226(22,6 \%)$ satisfatório (Figura 1) e das 774 situações de tratamento endodôntico insatisfatório $480(62,0 \%)$ apresentavam-se sem lesões periapicais e $294(38,0 \%)$ com lesões periapicais (Figura 2). Nos 226 casos de tratamento endodôntico satisfatório 48 $(21,2 \%)$ apresentavam-se com lesões periapicais e 178 $(78,8 \%)$ sem lesões periapicais (Figura 3 ).

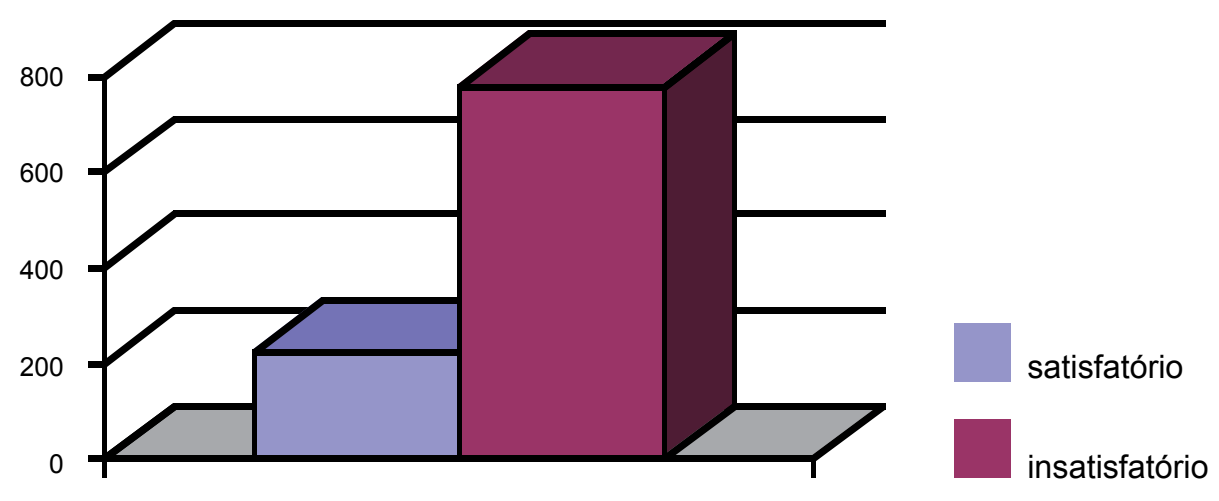

FIGURA 1 - Qualidade do tratamento endodôntico considerados satisfatórios. 
AVALIAÇÃO DAS CARACTERÍSTICAS DA CONTENÇÃO INTRA-RADICULAR E TRATAMENTOS ENDODÔNTICO EM RADIOGRAFIAS PERIAPICAIS DE 1000 DENTES

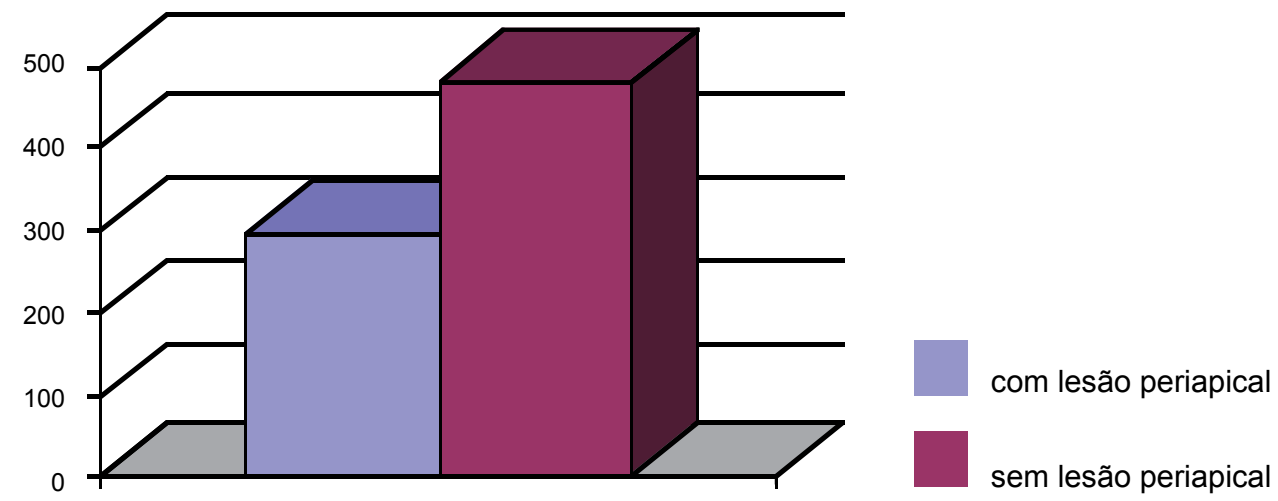

FIGURA 2 - Presença de lesão periapical em tratamentos endodônticos considerados insatisfatórios.
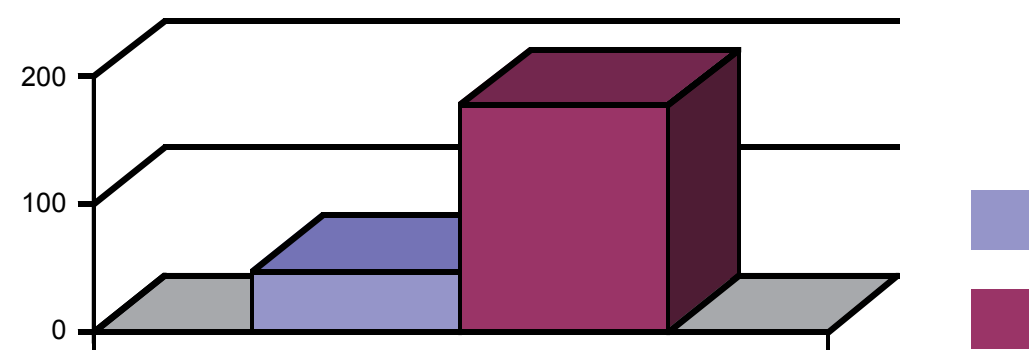

com lesão periapical

sem lesão periapical

FIGURA 3 - Presença de lesão periapical em tratamentos endodônticos considerados satisfatórios.

Em relação aos pinos, verificou-se que 106 $(10,6 \%)$ eram satisfatórios e $894(89,4 \%)$ insatisfatórios (Figura 4). Quanto à análise dos dois eventos pino e tratamento endodôntico, verificou-se que $40(4,0 \%)$ dos casos avaliados apresentavam-se em condições satisfatórias, $708(70,8)$ insatisfatórias e $252(25,25)$ combinações variadas (Figura 5).

Com o objetivo de verificar a existência ou não de diferenças significantes entre as freqüências de alterações e de não-alterações periapicais, encontradas nas radiografias, foi aplicado o teste do Qui-Quadrado ${ }^{26}$, considerando-se a qualidade do tratamento endodôntico e a qualidade dos pinos como satisfatórias ou insatisfatórias.
O nível de significância foi estabelecido em 0,05, em uma prova bilateral.

$\mathrm{O}$ valor do $\mathrm{X}^{2}$ crítico $=3,84$, para 1 grau de liberdade, de acordo com a tabela dos Valores Críticos do Qui-Quadrado ${ }^{26}$. (*) $\mathrm{p}<0,05$

De acordo com os resultados demonstrados na tabela 1, foram encontradas diferenças significantes com relação à qualidade do tratamento endodôntico, sendo que os tratamentos satisfatórios apresentaram menor freqüência de alterações periapicais do que os insatisfatórios. Com relação a qualidade dos pinos, não foram encontradas diferenças significantes entre as freqüências de presença ou ausência de alterações. 
AVALIAÇÃO DAS CARACTERÍSTICAS DA CONTENÇÃO INTRA-RADICULAR E TRATAMENTOS ENDODÔNTICO EM RADIOGRAFIAS PERIAPICAIS DE 1000 DENTES

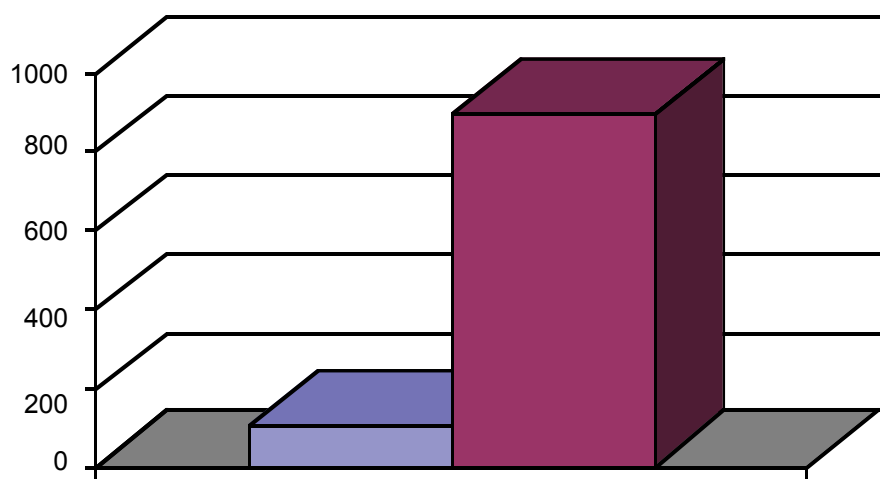

satisfatório

insatisfatório

FIGURA 4 - Qualidade da contenção intra-radicular.

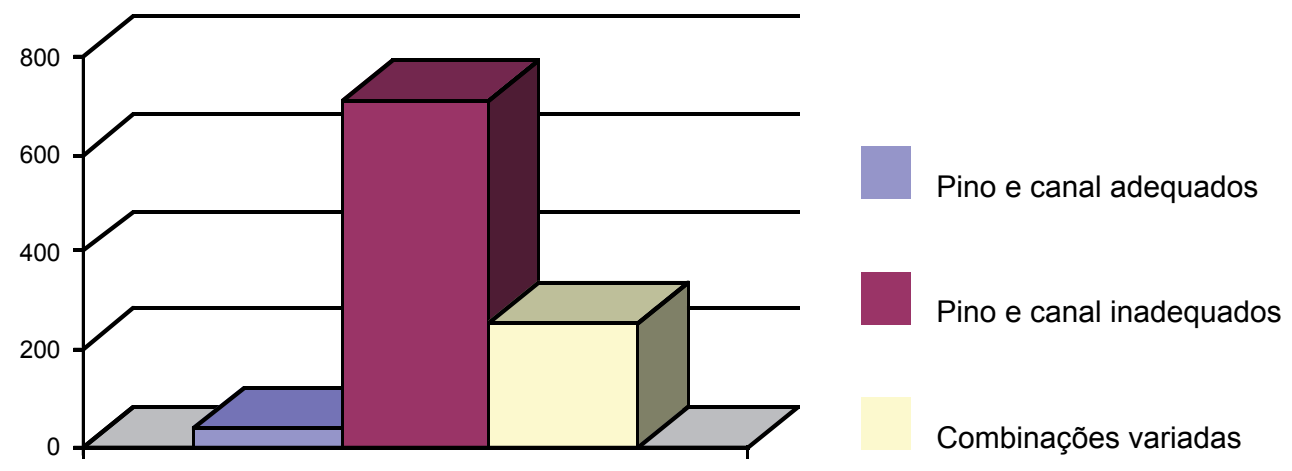

FIGURA 5 - Qualidade da contenção intra-radicular e tratamento endodôntico.

Tabela 1 - Valores do X2 encontrados, quando da aplicação do teste do Qui-Quadrado às freqüências de radiografias que apresentaram alterações periapicais ou não, considerando-se a qualidade do tratamento endodôntico e a qualidade dos pinos como satisfatório

\begin{tabular}{l|l}
\hline Variáveis Analisadas & Valores do X2 \\
\hline Tratamento Endodôntico & $21,80^{*}$ \\
Satisfatório x Insatisfatório x com alterações x sem alterações periapicais & \\
Pino & 0,35 \\
Satisfatório x Insatisfatório x com alterações x sem alterações periapicais & \\
\hline
\end{tabular}




\section{Discussão}

Todo processo restaurador realizado com coroa retida a pino em dente com tratamento endodôntico é um processo indissociável, ou seja, para se obter sucesso, todos os procedimentos devem ser realizados dentro de parâmetros bem definidos. O que pode ser visto na literatura em que vários trabalhos apontam de forma independente, que falhas no tratamento endodôntico ou na confecção do pino, realizados sem a observação dos princípios biológicos, estão fatalmente encaminhados ao insucesso. No tratamento endodôntico, a não remoção de resto pulpar, a falta de limpeza e desinfecção do canal radicular, a falha da condensação lateral durante a obturação do canal radicular, podem permitir a contaminação da região apical com fluídos bucais ${ }^{29}$, e as obturações, no limite apical inadequado, podem dar abrigo às bactérias, ou atuar como corpo estranho extravasado no periápice, permitindo que fiquem mais tempo na região apical, e comprometendo a saúde apical ${ }^{12}$. A persistência das bactérias na região apical pode dar origem ou transformar a reação inflamatória preexistente na região. Quando a população de bactérias é grande, vai haver uma agressão de alta intensidade e, como conseqüência, o aparecimento de uma reação inflamatória aguda de evolução rápida, em poucas horas, caracterizada por três sinais: calor; tumor e rubor e um sintoma, a dor. Quando a quantidade de bactérias é pequena, a agressão é de baixa intensidade, e a reação inflamatória é crônica, de evolução lenta, dias ou semanas, caracterizada pelos mesmos sinais e sintomas, mas de uma forma bastante atenuada, sendo, algumas vezes, de difícil identificação. Ainda, a persistência das bactérias e da reação inflamatória na região apical por um longo período, meses ou anos, permite a reabsorção óssea na região que passa a ser identificada nas tomadas radiográficas com imagem radiolúcida, que vai desde um espessamento do espaço periodontal apical até a uma grande área radiolúcida.

Do exposto acima, é possível inferir que a presença ou persistência de sintomatologia dolorosa espontânea ou provocada localizada na região apical e/ou a presença de alteração apical identificada por uma tomada radiográfica denunciam a possível presença de alterações em um dente com tratamento endodôntico.

A literatura tem demonstrado, de uma forma efetiva, a relação da saúde apical com alguns fatores, como: limite de obturação do canal radicular, que deve ser de $1 \mathrm{a} 2 \mathrm{~mm}$ aquém do ápice ${ }^{9,23,24}$; comprimento do material obturador remanescente, quando do preparo do canal para receber o pino deve ter no mínimo $3 \mathrm{~mm}^{3,5,21}$; necessidade de instalação coroas definitivas logo após tratamento endodôntico ${ }^{13,22}$; tratamento endodôntico incompleto $^{16,30}$ e presença de alterações periapicais pré-operatória são responsáveis pelo insucesso de um tratamento endodôntico ${ }^{8,29}$. Contudo não foi possível encontrar na literatura trabalhos que tenham demonstrado a relação direta entre pino cimentado/tratamento endodôntico e saúde apical, sendo assim, o presente estudo propõe uma avaliação radiográfica de dentes tratados endodonticamente com contenção intra-radicular, relacionando a presença de alterações apicais com a qualidade dos tratamentos endodônticos e contenções intra-radiculares. Ao final deste estudo encontrou-se por meio das análises das radiografias que, dos 1000 dentes examinados, $226(22,6 \%)$ apresentavam tratamento endodôntico satisfatório e $774(77,4 \%)$ insatisfatório. Estes resultados são semelhantes aos observados em outros trabalhos ${ }^{13,16,19}$.

Outro dado observado em nossa análise foi que, dos 774 tratamentos endodôntico insatisfatórios, 480 deles não tinham alterações apicais, representando $62,0 \%$ dos casos e, nos 226 tratamentos satisfatórios, 178 também não apresentaram alteração representando 78,7\%. Esses dados permitem concluir que os tratamentos insatisfatórios oferecem um risco maior de insucesso, da mesma forma demonstrado por Pesce, Bastos e Risso ${ }^{19}$ (1996), que, analisando o índice de ocorrência de retratamentos endodônticos em função da qualidade da obturação, observaram que $66,9 \%$ deles apresentavam restaurações incompletas e rarefação com indicação de retratamento. Kirkevang, Orstavik, Horsted-Blindslev ${ }^{13}$ (2000), analisando as condições periapicais com a qualidade da obturação radicular e restaurações de coroas de 733 dentes da população dinamarquesa, observaram que 64,0\% dos dentes apresentavam-se saudáveis, quando havia a adequada obturação do canal radicular. Tronstad et al. ${ }^{30}$ (2000), analisando radiograficamente 1001 dentes, tratados endodonticamente, observaram que $78,0 \%$ dos casos cujos tratamentos eram satisfatórios não apresentavam lesão.

Na presente pesquisa, $21,2 \%$ dos casos considerados endodonticamente satisfatórios apresentaram-se com lesões periapicais. Esse fato nos chamou a atenção e alguns questionamentos se tornam importantes no momento.

As lesões apicais observadas poderiam estar relacionadas com um processo de reparo parcial, pois não tivemos o controle inicial do tratamento. Poderíamos 
considerar que alguns desses dentes já apresentassem lesão periapical pré-operatória e que não houve resposta favorável ao tratamento endodôntico ${ }^{8,27,29}$. Uma outra questão que deve ser abordada é o fato da radiografia não ter evidenciado a plenitude do tratamento, já que as imagens radiográficas não são tridimensionais, podendo ocorrer erros no diagnóstico. De acordo com Maniglia, Biffi e Carvalho ${ }^{17}$ (1997); Biffi, Maniglia e Pascon $^{2}$ (1998), as falhas de condensação como também presença de remanescentes pulpares e detritos juntamente com o material obturador, encontradas principalmente na região apical, poderiam levar a uma contaminação do canal, se, durante o preparo para a instalação de um pino, não forem tomados os cuidados de assepsia e anti-sepsia obrigatórios durante os procedimentos endodônticos.

Outra hipótese a ser considerada para esse achado de dentes com aparente massa de obturação homogênea e lesão periapical seria uma contaminação durante o processo de preparação do pino de forma insatisfatória, não respeitando as normas necessárias para um procedimento sem contaminação, sem deslocamento dos cones e sem rompimento do cemento ou mesmo quando o pino é preparado e a restauração provisória é feita, demorando para a colocação de uma restauração definitiva, permitindo, assim, infiltrações indesejadas ${ }^{7,10}$. Trabalho como o de Raiden e Gendel$\operatorname{man}^{21}$ (1994), demonstra, em estudo "in vitro", que o preparo do canal não altera o selamento, porém, em ambos os relatos, podem-se observar o cuidado tido pelos pesquisadores durante o processo de preparação e obturação dos dentes, bem como a remoção do material que, além de ter sido feita de modo criterioso, foi preparado logo após obturação, o que justificaria tal afirmativa. Segundo $\operatorname{Prado}^{20}$ (2003) a permeabilidade da obturação no sentido cérvico-apical, é significativamente maior nos preparos dos espaços para pino feitos após presa do material, quando, comparados àqueles feitos imediatamente após a obturação do canal. Diante deste fato seria importante, que houvesse um trabalho conjunto entre protesista e endodontista, quando da indicação de um pino intra-radicular, para que o endodontista ficasse responsável pelo preparo e selamento do espaço logo após obturação do canal, evitando-se, assim, que canais radiculares considerados satisfatórios viessem a ser contaminados, provocando possíveis alterações periapicais. Em relação ao elevado número de dentes com tratamento endodôntico considerados insatisfatórios (774), e sendo que $480(62,0 \%)$ sem lesão apical, possa ser explicado por possível presença de um bom vedamento coronal presente e comprimento do remanescente dentro do limite de $3 \mathrm{~mm}$, que, nos achados, representaram $89,9 \%$ dos casos analisados. Ray e Trope ${ }^{22}$ (1995), utilizando radiografias periapicais, analisaram as condições periapicais de dentes tratados endodonticamente, tomando como base a qualidade técnica das obturações e as restaurações coronárias e observaram que a qualidade técnica das coroas teve maior significância na saúde apical, quando comparada com o tratamento endodôntico, A análise dos pinos mostrou que apenas $106(10,6 \%)$ satisfizeram às condições básicas para serem considerados satisfatórios, e 894 $(89,4 \%)$ foram considerados insatisfatórios. Na análise dos $106(10,6 \%)$ dentes com pinos satisfatórios, 67 $(63,2 \%)$ não apresentaram alteração periapical, e dos outros $894(89,4 \%)$ pinos, considerados insatisfatórios $591(66,1 \%)$ não apresentavam alteração periapical, o que demonstra não existir relação entre a qualidade do pino e a presença de alteração periapical. Tronstad et al. ${ }^{30}$ (2000) analisaram 1001 dentes tratados endodonticamente, sendo 528 com pino e, destes, 71,0\% não apresentaram alteração periapical e, dos $473 \mathrm{sem}$ pino, $64,0 \%$ não apresentavam alteração periapical, não havendo diferença estatística significante entre os dois grupos, permitindo aos autores concluíram que a presença de pinos não afeta negativamente os resultados de tratamentos endodônticos. Kvisk, Rydin e Reit ${ }^{14}$ (1989) estudaram a freqüência de lesões periapicais em 852 dentes tratados endodonticamente, sendo que $424 \mathrm{com}$ canais radiculares retidos a pino. Desse estudo, puderam concluir que a colocação do pino, por si só, não pode diminuir a probabilidade de cicatrização do ápice radicular ou a manutenção da sua saúde, porém um selamento radicular de, no mínimo $3 \mathrm{~mm}$, é importante para a saúde apical. Hommez, Cappens e De Morr ${ }^{11}$ (2002), em estudo clínico e radiográfico de 745 dentes, relacionando o tratado endodôntico e restaurações com presença de alteração apical, afirmaram que a presença de pino no canal radicular não tem influência na saúde apical. Contrariamente aos achados anteriores, Eckerbom, Magnusson e Martinsson ${ }^{6}$ (1991), após estudarem a prevalência de lesão apical em dentes tratados endodonticamente e coroados e retidos a pino em população sueca, concluíram que dentes com pinos têm mais periodontites apicais que outros dentes. Quando da análise dos dois eventos pino e tratamento endodôntico, observou-se que apenas $40(4,0 \%)$ dos dentes tinham pino/tratamento endodôntico satisfatórios, e, destes, $28(70,0 \%)$ não apresentaram alteração periapical e $708(70,8 \%)$ tinham pino/tratamento insatisfatórios e, destes, 441 
$(62,0 \%)$ não apresentaram alteração periapical, uma diferença de dados que não nos permite dizer se há uma relação entre sucesso de um tratamento e tratamentos insatisfatórios.

Neste estudo, não foi possível relacionar independentemente cada um dos critérios usados quanto à qualidade do tratamento endodôntico e características do pino como possíveis causas de insucesso do tratamento, já que um único dente apresentou duas ou mais falhas. Constatadas as falhas em um tratamento, caracterizadas pela presença de alteração periapical, é importante que o profissional faça o estudo de um possível retratamento, tendo bem claras as causas que levam ao seu sucesso ou insucesso. Os resultados encontrados neste estudo demonstraram uma incidência muito alta de fracasso dos tratamentos endodônticos em dentes restaurados com retentor intra-radicular, necessitando de uma avaliação do ensino para melhor entender a causa desses insucessos. Observação semelhante a estas foi feita por Lopes et al. ${ }^{16}$ (1997) quando da análise de 500 dentes tratados endodonticamente e restaurados com retentor intra-radicular para avaliar o comprimento dos pinos e a qualidade dos tratamentos endodônticos de pacientes que procuraram a Clínica de Endodontia da Policlínica Geral do Rio de Janeiro e da clínica particular dos autores.

É importante ressaltar que o exame radiográfico fica limitado por uma imagem estática, e somente terá o valor diagnóstico, quando relacionado ao clínico. Contudo algumas imagens podem sugerir uma possível alteração óssea na região apical, que, conceitualmente, poderia ser compatível com um quadro clínico. Nas reações inflamatórias crônicas, que se arrastam por um tempo maior, há tempo suficiente para que ocorra reabsorção óssea apical que pode ser identificada como uma imagem radiolúcida, ou seja, permite a passagem de maior quantidade de radiação. Essa imagem radiolúcida apical pode variar de tamanho, aparecendo como um espessamento do espaço periodontal apical até como uma grande área radiolúcida.

Os resultados da presente pesquisa nos levam a acreditar que o insucesso dos tratamentos endodôntico e/ou da contenção intra-radicular, estejam relacionados às dificuldades técnicas dos profissionais em realizarem um tratamento adequado.

\section{Conclusão}

Os resultados das análises radiográficas feitas no presente estudo permitiram-nos concluir que:

1. Dos 1000 dentes analisados radiograficamente, $77,4 \%$ tratamentos endodônticos e $88,45 \%$ das contenções intra-radiculares foram considerados insatisfatórios;

2. De acordo com análise estatística não-paramétrica $^{26}$, foram encontradas diferenças significantes com relação à qualidade do tratamento endodôntico, sendo que os tratamentos insatisfatórios apresentaram maior freqüência de alterações periapicais do que os satisfatórios;

3. Com relação à qualidade dos pinos, não foram encontradas diferenças significantes entre as freqüências de presença ou de ausência de alterações.

\begin{abstract}
The purpose of the present investigation was to evaluate the quality of the endodontic treatment and the post, and their relationship with apical periodontitis in 1000 teeth using periapical radiographs. The radiographs were digitalized and the analyses procedure were based on a satisfactory endodontic treatment when canals were homogeneously filled $2 \mathrm{~mm}$ from the radiographic apex and when was left apical filling of at least $3 \mathrm{~mm}$. The post was considered satisfactory when its length was 2/3 of the root length or the same length of the clinical crown and its diameter was 1/3 of the root diameter. The posts were qualified as unsatisfactory when radiographic images showed empty spaces between the post and the endodontic treatment, or there was post deviation from the root axis, or a root fracture. The existence of periapical lesion was admitted when an enlargement or a radiolucence of the periapical space were present. It can be concluded that there is a relationship statistically significant between teeth with unsatisfactory endodontic treatment and periapical lesions and there is not the same relationship between unsatisfatory post with periapical lesions.
\end{abstract}

\title{
UNITERMS
}

Endodontics - post and core technique. 


\section{REFERÊNCIAS}

1. Barbosa HG, Holland R, de Souza V, Dezan EJ, Bernabe PF, Otoboni $\mathrm{JA}$, et al. Healing process of dog teeth after post space preparation and exposition of the filling material to the oral environment. Braz Dent $\mathbf{J}$ 2003; 14(2):103-8.

2. Biffi JCG, Maniglia CAG, Pascon EA. Computadorized method for comparative morphometrical analysis of two root canal obturation techniques. Bras End J 1998; 3(2): 14-20.

3. Caputo AA, Standlee JP. Pins and posts--why, when and how. Dent Clin North Am. 1976 Apr.;20(2): 299-311.

4. De Deus, QD. Obturação do Canal Radicular. In: Endodontia. Rio de Janeiro: Medisi; 1986. p.377-79.

5. DeCleen MJ. The relationship between the root canal filling and post space preparation. Int Endod J. 1993 Jan; 26(1):53-8.

6. Eckerbom M, Magnusson T, Martinsson T. Prevalence of apical periodontitis, crowned teeth and teeth with posts in a Swedish population. Endod Dent Traumatol. 1991 Oct; 7(5):214-20.

7. Filho LG. Capacidade Seladora do remanescente de obturação do canal radicular frente à infiltração microbiana. [dissertação] Uberlândia: Faculdade de Odontologia de Uberlândia, Universidade Federal de Uberlândia; 2005.

8. Holland R, Valle GF, Taintor JF, Ingle JI. Influence of bony resorption on endodontic treatment. Oral Surg Oral Med Oral Pathol. 1983 Feb; 55(2):191-203

9. Holland R., Hizatugo R, Scarparo C. Avaliação radiográfica dos resultados obtidos com o tratamento endodôntico radical. Rev. Farm. Odont. 1971; 37(3): p.173-176, . apud Berger CR, Leonardo RT. Técnicas atuais de obturação dos canais radiculares. In: Cardoso RJA, Gonçalves, EAN. Endodontia-Trauma. São Paulo: Artes Médicas, 2002: 153-88.

10. Hollanda ACB. Avaliação da infiltração microbiana em obturações dos canais radiculares com cimentos resinosos. [dissertação]- Uberlândia: Faculdade de Odontologia de Uberlândia,; Universidade Federal de Uberlândia 2005.

11. Hommez GM, Coppens CR, De Moor RJ. Periapical health related to the quality of coronal restorations and root fillings. Int Endod J. 2002 Aug; 35(8):680-9.

12. Ingle JI. Exitos y fracsos en endodoncia. Rev Assoc Odontol Arg 1962; 50(2): 67-74. apud Pesce HF, Bastos EF, Risso VA. Avaliação do índice de ocorrência de retratamentos endodônticos em função da qualidade da obturação, presença ou não de rarefação periapical e sintomatologia. Rev Bras Odontol 1996 Jul/Ago; 53(4): 29-31.

13. Kirkevang LL, Orstavik D, Horsted-Bindslev P, Wenzel A. Periapical status and quality of root fillings and coronal restorations in a Danish population. Int Endod J. 2000 Nov;33(6):509-15.

14. Kvist T, Rydin E, Reit C. The relative frequency of periapical lesions in teeth with root canal-retained posts. J Endod. 1989 Dec;15(12):578-80.

15. Leonardo MR. Contribuição ao estudo da reparação apical e periapical pós tratamento de canais radiculares. [Tese] Araraquara:Faculdade de Farmácia e Odontologia de Araraquara, Universidade Estadual Paulista; 1973. apud Berger CR, Leonardo RT. Técnicas atuais de obturação dos canais radiculares. In: Cardoso RJA, Gonçalves, EAN. Endodontia-Trauma. São Paulo: Artes Médicas; 2002. $153-88$.
16. Lopes HP, Estrela, C, Rocha NSM, Costa Filho, AS, Siqueira Júnior, JF. Retentores intra-radiculares: análise radiográfica do comprimento do pino e da condição da obturação do canal radicular. Rev. Bras. Odontol. 1997 Set.-out; 54(5):277-80.

17. Maniglia CAG, Biffi JCG, Carvalho LAP. Aspecto da guta-percha após a obturação pela técnica de condensação lateral e ultra-sônica. Rev Assoc Paul Cir Dent 1977 jul/ago; 51(4): 325-30.

18. Morgano SM. Restoration of pulpless teeth: application of traditional principles in present and future contexts. J Prosthet Dent. 1996 Apr;75(4):375-80.

19. Pesce HF, Bastos EF, Risso VA. Avaliação do índice de ocorrência de retratamentos endodônticos em função da qualidade da obturação, presença ou não de rarefação periapical e sintomatologia. Rev Bras Odontol 1996 Jan-fev; 53(1): 29-31.

20. Prado CJ. Avaliação da permeabilidade e efeito antimicrobiano da obturação endodôntica remanescente, após o alívio do canal radicular. Ribeirão Preto; 2003. [tese]- Ribeirão Preto: Faculdade de Odontologia de Ribeirão Preto, Universidade de São Paulo; 2003.

21. Raiden GC, Gendelman H. Effect of dowel space preparation on the apical seal of root canal fillings. Endod Dent Traumatol. 1994 Jun;10(3):109-12.

22. Ray HA, Trope M. Periapical status of endodontically treated teeth in relation to the technical quality of the root filling and the coronal restoration. Int Endod J. 1995 Jan;28(1):12-8.

23. Ricucci D, Langeland K. Apical limit of root canal instrumentation and obturation, part 2. A histological study. Int Endod J. 1998 Nov;31(6):394-409.

24. Ricucci D. Apical limit of root canal instrumentation and obturation, part 1. Literature review. Int Endod J. 1998 Nov;31(6):384-93.

25. Selby A. Fixed prosthodontic failure. A review and discussion of important aspects. Aust Dent J. 1994 Jun;39(3):150-6.

26. Siegel S. Estatística não-paramétrica, para as ciências do comportamento. São Paulo: McGraw-Hill do Brasil; 1975.

27. Sjogren U, Hagglund B, Sundqvist G, Wing K. Factors affecting the long-term results of endodontic treatment. J Endod. 1990 Oct;16(10):498-504.

28. Stern N, Hirshfeld Z. Principles of preparing endodontically treated teeth for dowel and core restorations. J Prosthet Dent. 1973 Aug;30(2):162-5.

29. Tamburus JR. Pesquisa radiográfica dos sucessos e insucessos do tratamento endodôntico. Rev Assoc Paul Cir Dent 1983; 37(3): 234-40.

30. Tronstad L, Asbjornsen K, Doving L, Pedersen I, Eriksen HM. Influence of coronal restorations on the periapical health of endodontically treated teeth. Endod Dent Traumatol. 2000 Oct;16(5):218-21.

Recebido em: 30/06/06 Aprovado em: 30/07/07

e-mail: ilaluci@gmail.com Endereço: Rua Urucânia ${ }^{\circ} 477$ Bairro Vigilato Pereira Telefone: (34) 3236-5876 Fax: (34) 3236-5876 